\title{
Modeling split-TCP latency and buffering requirements in GEO satellite networks
}

\author{
M. Karaliopoulos, R. Tafazolli and B. Evans \\ Centre for Communication Systems Research \\ University of Surrey \\ Guildford, GU2 7XH, United Kingdom
}

\begin{abstract}
The paper addresses the TCP performance enhancing proxy techniques broadly deployed in wireless networks. Drawing on available models for TCP latency, we describe an analytical model for the latency and the buffer requirements related to the split-TCP mechanism. Although the model applicability is broad, we present and evaluate the model in the context of geostationary satellite networks, where buffering requirements may become more dramatic. Simulation results are compared with the analytical model estimates and show that the model captures the impact of various parameters affecting the dynamics of the component connections traversing the terrestrial and the satellite network.
\end{abstract}

Keywords - split connections; GEO satellite links; TCP

\section{INTRODUCTION}

TCP Performance Enhancing Proxies (TCPPEPs) are amongst the favorite methods of enhancing TCP performance over wireless links, although they are viewed with skepticism within the IETF community. The main concerns are related to the reliability and the security of data communications that use the proxy services [1]: TCPPEPs conflict with the use of IPsec, which is mandatory for IPv6 and is regarded by many members of the Internet community as the unique way to enforce security in the Internet. The implementation of numerous features for TCP performance enhancement over wireless networks via use of intermediate agents, presupposes the capability to retrieve information carried in transport and application layer headers; this is not possible with standard IPsec. Nevertheless, the performance gain achieved with TCPPEPs appears to be too significant to be sacrificed. The proposal of Multi-Layer IPsec [2] reflects the will of the wireless communications community to preserve the performance enhancing features of TCPPEPs without sacrificing the advantages of IPsec.

Split connections are only one of the potential features of TCPPEPs. A proxy agent at the border between the wired and the wireless network intercepts the connection set-up (SYN) and termination (FIN) packets and initiates another connection over the wireless link. The proxy prematurely acknowledges the arriving TCP segments, before they reach the receiver at the other end of the wireless link, thus allowing the TCP sender endpoint to open its window quickly and accelerate the transfer rate. In the same time, it caches TCP segments so that they are forwarded to the destination under the command of the protocol deployed over the wireless link. Integrated PEP implementations feature a single PEP component residing on a single node, whereas distributed PEP implementations consist of multiple PEP components that may be hosted at multiple network nodes surrounding the wireless links over which performance enhancement is targeted [1].

Split connections have been studied extensively as a solution for terrestrial wireless networks- see, for example [3] and references therein. The additional problem in the case of GEO satellite networks is the high link latency that introduces higher buffering requirements at the border node (gateway) between the wired and the satellite network. The required buffer space is highly dependent on the transport protocol deployed over the satellite link. This may be either a TCP variant enriched with optimizations related to satellite environments or an independent protocol. In [4], TCP SACK is combined with three options, namely window scaling, timestamps and larger initial window [5], to make up an enhanced TCP version that is deployed over the satellite link. The advantage of this approach is that the satellite component of the connection inherits the flow/congestion control mechanisms of TCP that are necessary in shared satellite environments. On the other hand, the departure from TCP features allows higher flexibility in the protocol optimization. In [6], the authors propose the Satellite Transport Protocol (STP) as the satellite component of the end-to-end connection. STP replaces the per-TCP segment(s) ACKs with periodic exchange of reports between sender and receiver, which results in significant savings in the reverse path (i.e., the path followed by ACK packets) [7].

In this paper, we focus on integrated PEP split connection implementations that deploy TCP over the satellite link (splitTCP). In section II, we present our model for the split-TCP latency, namely the overall time spent on a finite transfer carried out by a split-TCP implementation, and the buffer requirements at the intermediate host hosting the TCPPEP agent. In doing so, we -inevitably- reproduce part of the analysis in [8], since it is required for the model formulation. We evaluate our model and discuss the simulation results in section III, before concluding our paper in section IV.

\section{THE MODEL}

\section{A. Split-TCP latency model}

Our starting point is the TCP latency model described by Cardwell, Savage and Anderson in [8]. The model can be 
considered an improvement over earlier models addressing only the congestion avoidance phase of TCP [9], [10], the applicability of which was limited to persistent TCP connections. It addresses connections that transfer a finite amount of bytes $D$, catering for the slow-start phase and the first loss recovery, and captures the impact of several protocol parameters such as the initial window $W^{\text {in }}$, the TCP delayed acknowledgment option and the maximum TCP receive window limitations $W^{\max }$. On the other hand, it invokes the model in [9] for the estimation of the steady-state throughput, hence inheriting its weaknesses: no modeling of the TCP fast recovery algorithm, sensitivity on precise knowledge of the connection round-trip time (RTT) and TCP retransmission timeout variable [11, chapter 3].

1) Terrestrial component of the split-TCP connection: Let $p_{T}$ be the TCP segment loss rate in the terrestrial component of the connection. This loss rate may be due either to congestion at the network buffers or link errors ${ }^{1}$. Following [8], the transfer evolves generally in three phases. The duration, or even relevance, of each phase to the transfer progress depends mainly on the encountered loss rate.

Without loss of generality, let us assume that the data transfer begins at time $t=0$. The expected number of TCP segments delivered from the terrestrial connection till the first packet loss is given in [8]:

$$
E\left[D_{T}^{s s}\right]= \begin{cases}D_{T}^{s g m} & \text { if } p_{T}=0 \\ \left(\frac{\left(1-\left(1-p_{T}\right)^{D_{T}^{s g m}}\right) \cdot\left(1-p_{T}\right)}{p_{T}}+1\right) & \text { if } p_{T}>0\end{cases}
$$

where $D_{T}^{s g m}=\left\lceil\frac{D}{M S S_{T}}\right\rceil$ is the transfer size in segments respectively and $M S S_{T}$ is the TCP segment size used by the terrestrial TCP sender. Unless otherwise noted, all window values mentioned subsequently imply TCP segments.

Let $W_{T}^{i n}$ be the initial window of the terrestrial connection and $\gamma_{T}$ be the rate of the congestion window ( $c w n d$ ) exponential growth during slow start, which is 2 if delayed acknowledgments are not activated and 1.5 otherwise. Had no constraint $W_{T}^{\max }$ been imposed due to the maximum TCP buffer size or the receive window, the mean cwnd size at the time of first packet loss would be

$$
E\left[W_{T}^{s s}\right]=\frac{E\left[D_{T}^{s s}\right] \cdot\left(\gamma_{T}-1\right)}{\gamma_{T}}+\frac{W_{T}^{i n}}{\gamma_{T}}
$$

Depending on the relation between $E\left[W_{T}^{s s}\right]$ and $W_{T}^{\max }$, two scenarios are possible. When $E\left[W_{T}^{s s}\right] \leq W_{T}^{\max }$, the slow-start phase is terminated at time

$$
t_{1}^{T}=R T T_{T} \cdot \log _{\gamma_{T}}\left(E\left[W_{T}^{s s}\right]\right)
$$

\footnotetext{
${ }^{1}$ The link error probability is independent of the congestion loss. The opposite does not hold in general.
}

during which the connection transfers $E\left[D_{T}^{s s}\right]$ segments. Otherwise, slow-start evolves in two sub-phases. The first one is terminated when the window reaches $W_{T}^{s s}$ at time

$$
t_{1 a}^{T}=R T T_{T} \cdot\left(\log _{\gamma_{T}}\left(\frac{W_{T}^{\max }}{\gamma_{T}}\right)+1\right)
$$

during which the connection transfers $D_{T}^{1 a}=\frac{\gamma_{T} \cdot W_{T}^{\max }-W_{T}^{i n}}{\gamma_{T}-1}$ segments. The second involves the transmission of $W_{T}^{\max }$ segments per round and completes the transmission of $E\left[D_{T}^{s s}\right]$ segments at time

$$
t_{1}^{T}=t_{1 a}^{T}+R T T_{T} \cdot \frac{\left(E\left[D_{T}^{s s}\right]-D_{T}^{1 a}\right)}{W_{T}^{\max }}
$$

The slow-start phase will be terminated upon a segment loss with probability

$$
l_{T}^{s s}=1-\left(1-p_{T}\right)^{D_{T}^{s g m}}
$$

leading to a retransmission timeout with probability [9]

$$
p_{\text {RTO }}=\min \left(1, \frac{1+\left(1-p_{T}\right)^{3} \cdot\left(1-\left(1-p_{T}\right)^{E\left[W_{T}^{s s}\right]-3}\right)}{\left(1-\left(1-p_{T}\right)^{E\left[W_{T}^{s s}\right.}\right) /\left(1-\left(1-p_{T}\right)^{3}\right)}\right) \text {. }
$$

When slow-start ends with a timeout, the suffered delay will be equal to

$$
T_{R T O}=T_{T}^{o} \cdot \frac{\left(1+p_{T}+2 \cdot p_{T}^{2}+4 \cdot p_{T}^{3}+8 \cdot p_{T}^{4}+16 \cdot p_{T}^{5}+32 \cdot p_{T}^{6}\right)}{1-p_{T}}
$$

where $T_{T}^{o}$ is the base TCP retransmission timer timeout value.

With probability $1-p_{R T O}$ the connection will enter Fast Recovery, whose duration is taken to be one round-trip time. Overall the connection will recover from the first segment loss in time

$$
t_{2}^{T}=t_{1}^{T}+l_{T}^{s S} \cdot\left(p_{R T O} \cdot T_{R T O}+\left(1-p_{R T O}\right) \cdot R T T_{T}\right) .
$$

The connection then proceeds in congestion avoidance mode, with a segment send rate $R_{T}$ derived in the steady-state throughput analysis in [9] and referred to in [8, Eq. 22]. The connection finally completes the transfer of $D$ bytes in time:

$$
t_{3}^{T}=t_{2}^{T}+\frac{\left(D_{T}^{s g m}-E\left[D_{T}^{s s}\right]\right)}{R_{T}}
$$

Therefore, the progress of the data transfer in bytes with time at the terrestrial component of the connection, $D_{T}(t)$, is summarized by (10).

2) Satellite component of the split-TCP connection: Assuming that the initial connection set-up messages are let pass through the network end-to-end, the data transfer over the satellite link begins with a hysteresis equal to $R T T_{T} / 2$.

Forgetting for a moment the dependence on the terrestrial TCP connection, the satellite component tends to evolve in a similar manner. Therefore we can estimate $E\left[D_{S}^{s s}\right], E\left[W_{S}^{s s}\right]$ as in equations (1) and (2) on the basis of $W_{S}^{i n}, \gamma_{S}, W_{S}^{\max }$, 


$$
\begin{aligned}
& D_{T}(t)=\left\{\begin{array}{cl}
M S S_{T} \cdot W_{T}^{i n} \cdot \frac{\gamma_{T}\left[\frac{t}{R T T_{T}}\right]-1}{\gamma_{T}-1} & 0 \leq t \leq t_{1 a}^{T}, \text { if } E\left[W_{T}^{s s}\right]>W_{T}^{\max } \\
D_{T}^{1 a}+\min \left(M S S_{T} \cdot W_{T}^{\max } \cdot\left[\frac{t-t_{1 a}^{T}}{R T T_{T}}\right], D\right) & t_{1 a}^{T} \leq t \leq t_{1}^{T}, \text { if } E\left[W_{T}^{s s}\right]>W_{T}^{\max } \\
M S S_{T} \cdot W_{T}^{i n} \cdot \frac{\gamma_{T}\left[\frac{t}{R T T_{T}}\right]-1}{\gamma_{T}-1} & 0 \leq t \leq t_{1}^{T}, \text { if } E\left[W_{T}^{s s}\right] \leq W_{T}^{\max } \\
M S S_{T} \cdot E\left[D_{T}^{s s}\right] & t_{1}^{T} \leq t \leq t_{2}^{T} \\
E\left[D_{T}^{s s}\right]+M S S_{T} \cdot R_{T} \cdot\left(t-t_{2}^{T}\right) & t_{2}^{T} \leq t \leq t_{3}^{T}
\end{array}\right. \\
& D_{S}(t)=\left\{\begin{array}{cl}
M S S_{S} \cdot W_{S}^{i n} \cdot \frac{\left.\gamma_{S} \frac{t-R T T_{T} / 2}{R T T_{S}}\right]-1}{\gamma_{S}-1} & \frac{R T T_{T}}{2} \leq t \leq \hat{t}_{1 a}^{S}, \text { if } E\left[W_{S}^{s s}\right]>W_{S}^{\max }, \\
D_{S}^{1 a}+\min \left(M S S_{S} \cdot W_{S}^{\max } \cdot\left[\frac{t-\hat{t}_{1 a}^{S}}{R T T_{S}}\right], D\right) & \hat{t}_{1 a}^{S} \leq t \leq \hat{t}_{1}^{S}, \text { if } E\left[W_{S}^{s s}\right]>W_{S}^{\max } \\
M S S_{S} \cdot W_{S}^{i n} \cdot \frac{\left.\gamma_{S} \frac{t-R T_{T} / 2}{R T T_{S}}\right]-1}{\gamma^{S}-1} & \frac{R T T_{S}}{2} \leq t \leq \hat{t}_{1}^{S}, \text { if } E\left[W_{S}^{s s}\right] \leq W_{S}^{\max } \\
M S S_{S} \cdot E\left[D_{S}^{s s}\right] & \hat{t}_{1}^{S} \leq t \leq \hat{t}_{2}^{S} \\
E\left[D_{S}^{s s}\right]+M S S_{S} \cdot R_{S} \cdot\left(t-\hat{t}_{2}^{S}\right) & \hat{t}_{2}^{S} \leq t \leq \hat{t}_{3}^{S}
\end{array}\right.
\end{aligned}
$$

$M S S_{S}, R T T_{S}$, and if we add a constant time shift to the respective time instants

$$
\hat{t}_{x}^{S}=t_{x}^{S}+\frac{R T T_{T}}{2}, x \in\left\{\begin{array}{llll}
1 & 1 & a & 2
\end{array}\right\},
$$

the cumulative number of bytes sent by the satellite component of the end-to-end connection is given by (11).

\section{B. Latency}

Depending on the relation between $D_{T}(t), D_{S}(t)$ we can then identify two cases:

a) $D_{T}(t) \geq D_{S}(t) \quad \forall t \in\left[\frac{R T T_{T}}{2}, t_{3}^{T}\right]$

In this case there are always TCP segments in the gateway buffers and the satellite component of the connection never starves. The overall transfer latency is dictated by the satellite component of the connection that evolves more slowly than its terrestrial counterpart

$$
E\left[T_{\text {split }}\right]=\hat{t}_{3}^{S}
$$

b) $\exists t^{\prime}:\left\{D_{T}\left(t^{\prime}\right)<D_{S}\left(t^{\prime}\right) \quad \frac{R T T_{T}}{2} \leq t^{\prime} \leq t_{3}^{T}\right\}$

In this case there is at least one interval during the transfer completion, where the gateway buffers empty. Therefore the TCP performance over the satellite link is actually constrained by the terrestrial portion of the connection. The overall transfer latency is now:

$$
E\left[T_{\text {split }}\right]=t_{3}^{T}+R T T_{S}
$$

\section{Buffer requirements}

Apparently, the buffered data $B(t)$ at the gateway are given by

$$
B(t)=\left\lceil D_{T}(t)-D_{S}(t)\right\rceil^{+}
$$

where $\lceil x\rceil^{+}$denotes the minimum non-negative integer number that is equal to or larger than $\mathrm{x}$.

\section{Model Evaluation}

We evaluate the model accuracy against ns2 [12] simulations. The simulation scenario is depicted in Figure 1: a single TCPPEP 


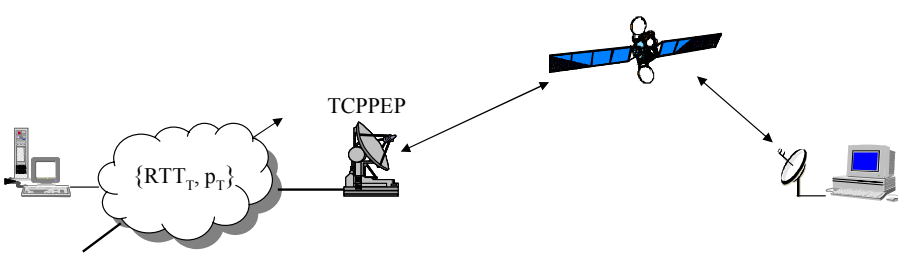

Figure 1. Simulation scenario for the validation of the split-TCP model agent, located at the satellite gateway node, splits a TCP connection between a server node at the terrestrial network and an end node behind a satellite terminal.

We perform file transfers ranging from $20 \mathrm{kB}$ to $2 \mathrm{MB}$ varying the quality of the satellite link, the TCP round-trip time and the packet loss faced by the terrestrial component of the connection. The default satellite link capacity (data rate) is $512 \mathrm{~kb} / \mathrm{s}$. We used the TCP NewReno variant [13] with the timestamps option activated. $W^{\max }$ is set to $32 \mathrm{kB}$, which is an intermediate value between the default settings for Windows $2000(16 \mathrm{kB})$ and the Windows XP $(64 \mathrm{kB})$, unless otherwise stated. The TCP MSS is 526 bytes allowing for the timestamps option (10 bytes) and 40 bytes TCP/IP headers. The granularity of the TCP timer is $100 \mathrm{~ms}$ and the initial window is one TCP segment.

For all simulation results shown in the rest of the paper, we plot mean values, estimated as the average of 50 simulation runs, along with their $95 \%$ confidence intervals.

\section{A. TCP latency}

1) No packet loss at the wired network: In this case, the splitTCP latency is dictated by the dynamics of the satellite component of the end-to-end connection. Figure 2. suggests that as far as no packet loss is met at the terrestrial networks, the $R T T_{T}$ does not affect the overall latency, since the terrestrial component of the connection is much faster than its satellite counterpart The terrestrial component features a smaller, errorfree loop and its throughput is only constrained by the receive window $W_{T}^{\max }(32 \mathrm{kB})$. The link errors start having an impact on the overall latency after some transfer size-dependent value, ranging from $B E R=10^{-6}$ for $20 \mathrm{kB}$ transfers to $10^{-7}$ for $2 \mathrm{MB}$ transfers.

The model predictions follow closely the simulation outcome. The precision of the model increases with larger transfer sizes and higher error rates, whereas it is slightly optimistic at lower error rates. This is attributed to the non-negligible transmission (serialization) delay and the consequent queuing delay at the satellite link buffers. For a $512 \mathrm{~kb} / \mathrm{s}$ satellite link, this delay is not negligible ${ }^{2}$.

2) Packet loss at the terrestrial leg of the connection: We perform two sets of simulation experiments. In both sets we vary the packet loss rate $p_{T}$ at the terrestrial network, whereas we maintain fixed BER over the satellite link. In Figure 2a, the BER is set to the rather pessimistic value of $10^{-5}$. Although the throughput of the terrestrial connection is reduced, the overall

\footnotetext{
${ }^{2}$ Note that the authors in [8] validate their TCP latency model simulating transfers over a $1 \mathrm{Gbps}$ link, circumventing this way the impact of transmission and queuing delays.
}

split-TCP latency is still determined by the latency of the satellite component of the connection, since the high BER prevents TCP from reaching full speed over the satellite link. Only for $p_{T}$ as high as 0.2 is there notable performance deterioration suggesting that we enter the $\left\{p_{T}, p_{S}\right\}$ regime, where (15) holds. On the contrary, Figure $2 \mathrm{~b}$ plots the split-TCP latency when the BER is set to $10^{-8}$. Now, the terrestrial component of the connection appears to be the slower one and it is its own speed that defines the overall latency, according to (16).

Both figures basically confirm the robustness of the model to the dynamics of the terrestrial portion of the connection but also reveal the original TCP latency model inaccuracy with respect to the timeout value parameter $T_{o}$. This becomes obvious in the $B E R=10^{-8}$ case, where the overall latency is determined by the dynamics of the terrestrial connection. The probability of a timeout increases with $p_{T}$ along with the weight of the respective delay on the overall latency. The small $R T T_{T}$ makes the model predictions more sensitive to the $T_{o}$ setting, which is static in the latency model of [8] as it is static in the PFTK formula [9] that is incorporated in the former model. On the contrary, in the real protocol operation, $T_{o}$ is adjusted continuously according to the experienced RTT. The default value of $1 \mathrm{~s}$, chosen for these runs according to the suggestions in [8] and [9], appears to be reasonably good for high packet loss rates but too high for moderate error rates, as intuitively expected. Note that this inefficiency is hidden in Figure 3a, since the split-TCP latency is determined by the slower satellite component of the connection, which has a higher RTT.

\section{B. Buffer requirements}

In Figure $4 a-b$ we plot percentiles of the buffer requirements at the satellite gateway node hosting the TCPPEP. The buffered data were logged during simulation with a time step equal to $10 \mathrm{~ms}$, whereas the respective model values were obtained via sampling of (17) with the same frequency. The median $\left(50^{\text {th }}\right)$, the $90^{\text {th }}$ and the $99^{\text {th }}$ percentiles of the two data sets were obtained subsequently via off-line statistical processing of the derived time series. As it can also be seen in Table I, the agreement of the model with the simulation outcome improves with greater percentiles, which notably are the ones that matter in the estimation of buffer requirements. In particular, their $99^{\text {th }}$ percentile is a good estimate of the maximum buffer space that is required if data loss is to be avoided.

A closer look at the way the satellite gateway node buffer occupation evolves with time is given in Figure 5a and Figure $5 \mathrm{~b}$, which plot $D_{T}(t), D_{S}(t)$ and $B(t)$ according to (10), (11) and (17), respectively. The increase of error rate over the satellite link drives the satellite component of the connection earlier into the congestion avoidance phase and lengthens the data transfer over the satellite link. As a result, higher amounts of data have to stay longer at the buffers. Since no packet loss is encountered at the terrestrial network, the higher percentiles approximate the size of the transfer. 
a) $R T T_{T}=40 \mathrm{~ms}, p_{T}=0$

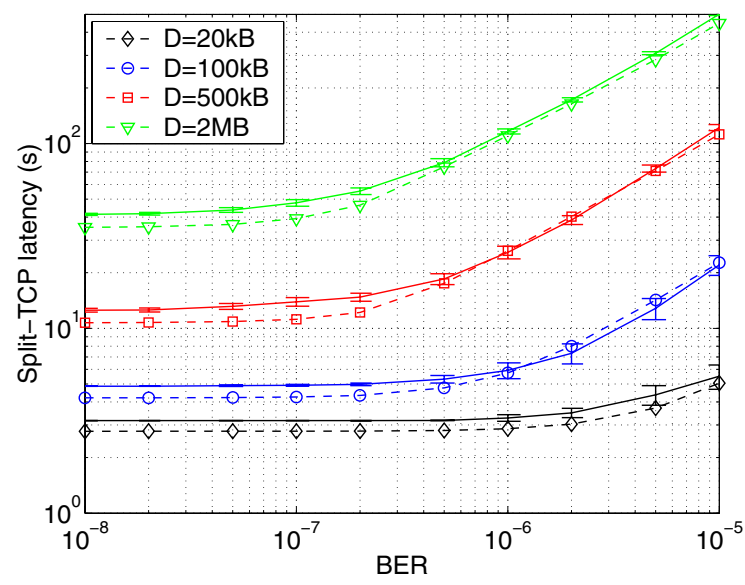

b) $R T T_{T}=200 \mathrm{~ms}, p_{T}=0$

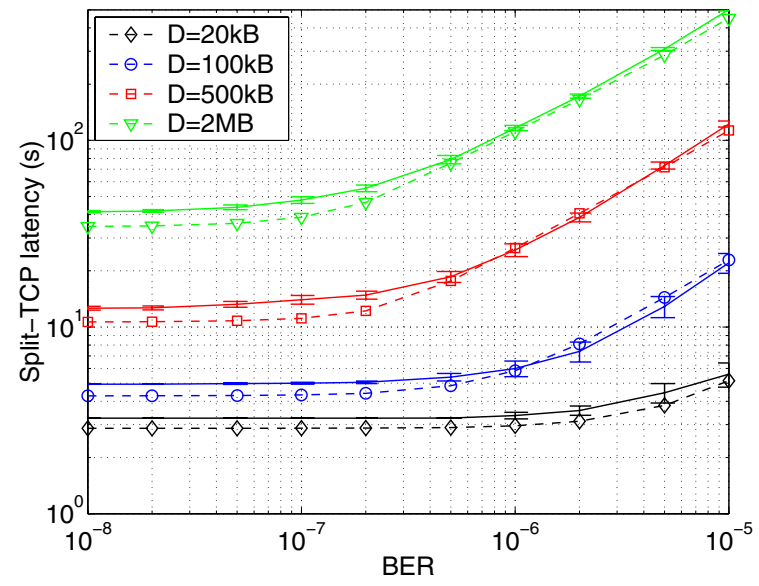

Figure 2. Split-TCP latency vs BER over the satellite link for various transfer sizes (dashed lines correspond to simulations)

a) $R T T_{T}=40 \mathrm{~ms}, \mathrm{BER}=10^{-5}$

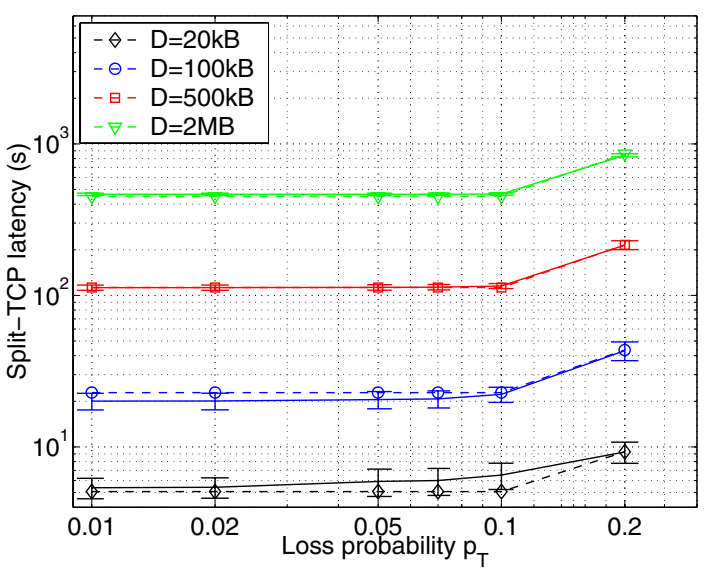

b) $R T T_{T}=40 \mathrm{~ms}, \mathrm{BER}=10^{-8}$

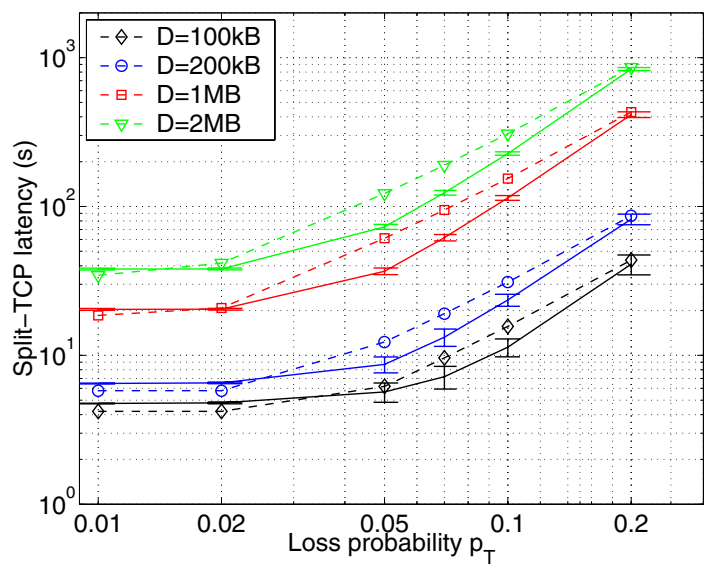

Figure 3. Split-TCP latency vs. packet loss at the terrestrial network (dashed lines correspond to simulations) $\curvearrowright-D=20 \mathrm{kB} \quad-\mathrm{D}=100 \mathrm{kB}$ $\mathrm{D}=500 \mathrm{kB} \quad \nabla-\mathrm{D}=2 \mathrm{MB}$

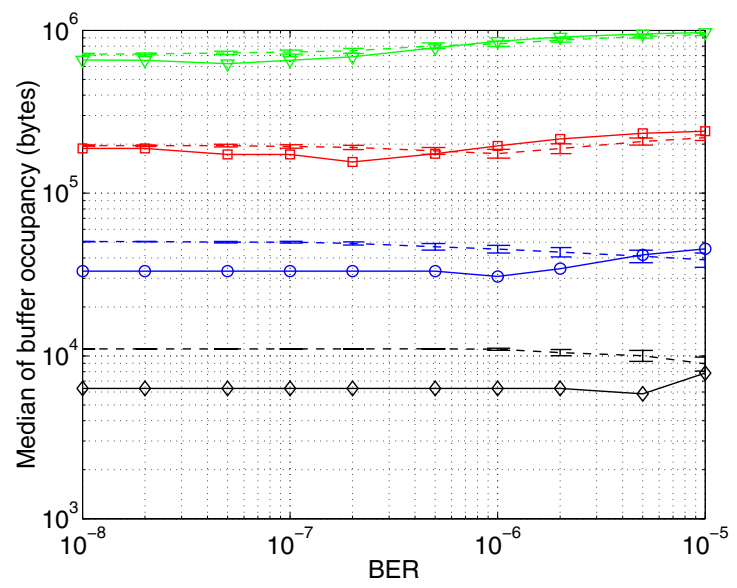

a) Median

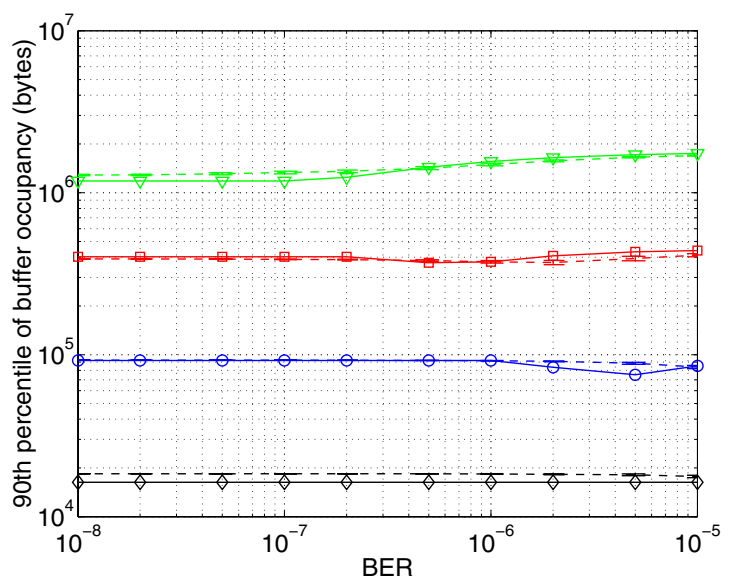

b) $90^{\text {th }}$ percentile

Figure 4. Statistics of buffer requirements vs BER over the satellite link for $\mathrm{RTT}_{\mathrm{T}}=200 \mathrm{~ms}$ and $\mathrm{p}_{\mathrm{T}}=0$ (dashed lines correspond to simulations) 


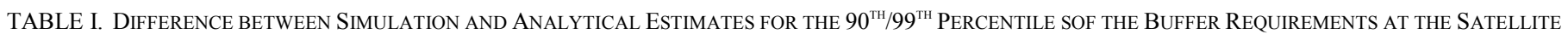
Gateway Node as a Percentage of the Simulation Estimates $\left(\mathrm{RTT}_{\mathrm{T}}=200 \mathrm{MS}, \mathrm{P}_{\mathrm{T}}=0\right)$

\begin{tabular}{|c|c|c|c|c|c|c|c|c|c|c|c|}
\hline & & \multicolumn{10}{|c|}{ BER over the satellite link } \\
\hline & & $10^{-8}$ & $2 \times 10^{-8}$ & $5 \times 10^{-8}$ & $10^{-7}$ & $2 \times 10^{-7}$ & $5 \times 10^{-7}$ & $10^{-6}$ & $2 \times 10^{-6}$ & $5 \times 10^{-6}$ & $10^{-5}$ \\
\hline \multirow{2}{*}{$\begin{array}{c}\text { Transfer } \\
\text { size }\end{array}$} & $20 \mathrm{kB}$ & $11.3 / 2.9$ & $11.3 / 2.9$ & $11.3 / 2.9$ & $11.3 / 2.9$ & $11.3 / 2.9$ & $11.3 / 2.9$ & $11.1 / 2.9$ & $10.7 / 2.9$ & $10 / 2.5$ & $7.9 / 2.5$ \\
\hline & $2 M B$ & $8.2 / 2.6$ & $8.3 / 2.8$ & $9.8 / 4.3$ & $11.4 / 6.1$ & $8.2 / 6.4$ & $1.2 / 2.8$ & $4.6 / 4.5$ & $4.7 / 4.2$ & $3.6 / 3.6$ & $3.1 / 2.9$ \\
\hline
\end{tabular}

a) $R T T_{T}=40 \mathrm{~ms}, \mathrm{BER}=10^{-6}$

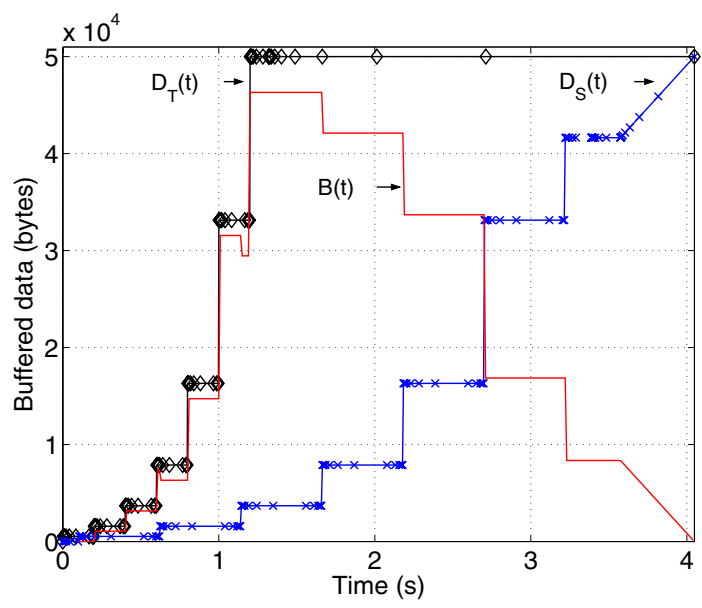

b) $R T T_{T}=40 \mathrm{~ms}, B E R=10^{-5}$

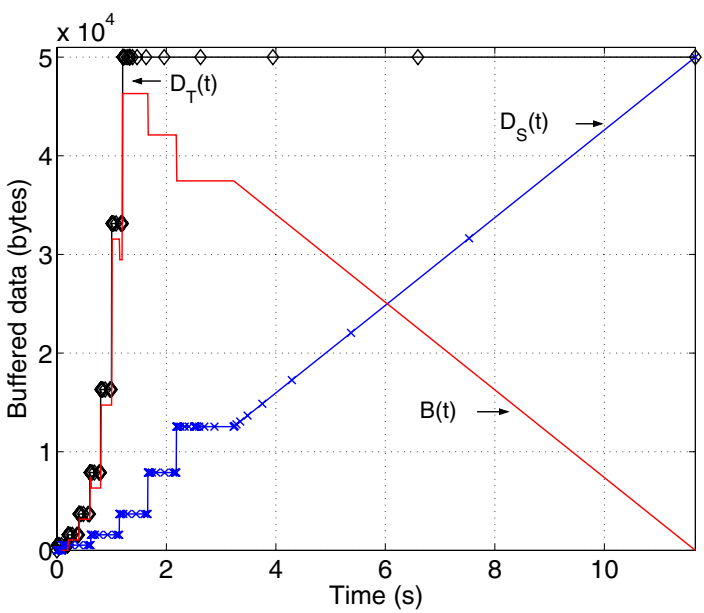

Figure 5. Cumulative transferred data from the terrestrial, $D_{T}(t)$, and the satellite, $D_{S}(t)$, components of the split connection and buffer occupation, $B(t)$, at the intermediate node hosting the proxy

\section{CONCLUSIONS}

We have presented a model for the latency of the split-TCP connection and the buffer requirements at the intermediate node, where splitting is deployed. The model draws on an existing model for TCP latency and inherits its advantages and drawbacks. Therefore, the model can capture the impact of slowstart and various TCP options deployed in the two components of the end-to-end connection. On the other hand, its estimates exhibit sensitivity to the (static) value chosen for the retransmission timeout. The comparisons of the model estimates with simulation results show good match between the two.

Notably the model provides a generic systematic approach for the estimation of the latency and buffer requirements related to the split connection mechanism. It can be adapted to accommodate split connection implementations that deploy different transport protocols over the satellite link, as long as analytical models for these protocols are available. Our current research evolves around this task.

\section{REFERENCES}

[1] J. Border et al., "Performance enhancing proxies intended to mitigate linkrelated degradations," Internet RFC 3135, June 2001

[2] Y. Zhang, "A Multi-Layer IP Security Protocol for TCP Performance Enhancement in Wireless Networks," IEEE Journal on Selected Areas in Communications, vol. 22, no. 4, pp. 767-776, May 2004

[3] H. Balakrishnan, V.N. Padmanabhan, S. Seshan and R.H. Katz, "A comparison of mechanisms for improving TCP performance over wireless link," IEEE/ACM Transactions on Networking, December 1997
[4] V.G. Bharadwaj, J.S. Baras and N.P. Butts, "An architecture for Internet service via broadband satellite networks," International Journal of Satellite Communications, vol. 19, no. 1, pp. 29-50, 2001

[5] M. Allman et al., "Ongoing TCP research related to satellites," Internet RFC 2760 , February 2000

[6] T. Henderson and R.H. Katz, "Satellite Transport Protocol (STP): An SSCOP-based transport protocol for datagram satellite networks," in Proc. 2nd International Workshop on Satellite-based Information Services (WOSBIS '97), Budapest, Hungary, Oct. 1997

[7] T. Henderson, "Transport protocols for Internet-compatible satellite networks," IEEE Journal of Selected Areas in Communications, vol. 17, no. 2, pp. 326-344, Feb. 1999

[8] N. Cardwell, S. Savage and T. Anderson, "Modelling TCP latency," in Proc. IEEE INFOCOM, Tel-Aviv, Israel, March 2000

[9] J. Padhye, V. Firoiu, D. Towsley and J. Kurose, "Modelling TCP Reno performance: a simple model and its empirical validation," ACM Computer Communications Review, vol. 28, no. 4, pp. 303-314, 1998

[10] M. Mathis, J. Semke, J. Mahdavi and T. Ott, "The macroscopic behaviour of the TCP congestion avoidance algorithm," Computer Communications Review, vol. 3, July 1999

[11]M. Karaliopoulos, "Support of elastic TCP traffic over GEO broadband satellite networks", PhD Thesis, University of Surrey, April 2004

[12] K. Fall and K. Varadhan, "Satellite networking in ns," the ns manual-chapter 17 , February 2002

[13] S. Floyd and T. Henderson, "The NewReno modification to TCP's Fast Recovery algorithm," Internet RFC 2582, April 1999 